\title{
A SHOOTING METHOD FOR SINGULAR NONLINEAR SECOND ORDER VOLTERRA INTEGRO-DIFFERENTIAL EQUATIONS
}

\author{
R. E. SHAW and L. E. GAREY \\ University of New Brunswick \\ Department of Mathematics, Statistics and Computer Science \\ Saint John, New Brunswick, Canada E2L 4L5
}

(Received August 30, 1995 and in revised form March 8, 1996)

ABSTRACT. The method of parallel shooting will be employed to solve nonlinear second order singular Volterra integro-differential equations with two point boundary conditions.

KEY WORDS AND PHRASES: Volterra integro-differential equation, boundary value problem, singular equation, numerical solution.

\section{AMS SUBJECT CLASSIFICATION CODES:}

\section{INTRODUCTION}

The form of the equation to be considered is given by

$$
y^{\prime \prime}=f(x, y, z), \quad 0 \leq x \leq a
$$

where

$$
z(x)=\int_{0}^{x} K(x, t, y(t)) d t .
$$

The function $y(x)$ denotes the solution and is unknown. Let $R_{1}$ and $R_{2}$ be two sets with

$$
R_{1}=((x, t, y): 0 \leq t \leq x \leq a,|y|<\infty)
$$

and

$$
R_{2}=((x, y, z): 0 \leq x \leq a,|y|<\infty,|z|<\infty) .
$$

The problem (1) is completed by adding the boundary conditions $y(0)=A$ and $y(a)=B$. Under the following assumptions.

i) $f$ and $K$ are uniformly continuous in each variable,

ii) for the function $f$ and for all $(x, y, z),(x, \bar{y}, z)$ and $(x, y, \bar{y}) \in R_{2}$

$$
\begin{aligned}
& |f(x, y, z)-f(x, \bar{y}, z)| \leq L_{1}|y-\bar{y}|, \\
& |f(x, y, z)-f(x, y, \bar{z})| \leq L_{2}|z-\bar{z}|
\end{aligned}
$$

iii) for the function $K$ and for all points $(x, t, y)$ and $(x, t, \bar{y}) \in R_{1}$

$$
|K(x, t, y)-K(x, t, \bar{y})| \leq L_{3}|y-\bar{y}|
$$

iv) the functions $f_{y} \geq 0, f_{z} \geq 0$ and $K_{y} \geq 0$ for all $(x, t, y) \epsilon R_{1}$ and $(x, y, z) \epsilon R_{2}$

the solution is unique. In [3], the form of the kernel function allowed for a finite number of singularities in the range of integration. Here we consider a nonlinear kernel of the form

$$
K(x, t, y(t))=S(x, t) G(t, y(t))
$$

where $S(x, t)$ contains the singularities associated with the function $K$. Following the work in [7], it is 
assumed that $S(x, t)$ satisfies:

i)

$$
\int_{0}^{x}|S(x, t)| d t \leq L<\infty, \text { for all } 0 \leq x \leq a
$$

and

ii)

for every $\epsilon>0$ there exists a $\delta=\delta(\epsilon)>0$ independent of $x$ and $\alpha$ such that

$$
\begin{aligned}
& \int_{\alpha}^{\alpha+\delta}|S(x, t)| d t<\epsilon \\
& \text { for all } \alpha \text { such that } 0 \leq \alpha \leq x-\delta .
\end{aligned}
$$

With these additional conditions, the singular problem has a unique solution. Boundary valued problems for ordinary differential equations have been considered by many authors. Two textbooks containing material on numerical solutions are given in the references [6,8]. References $[7,9,10]$ consider some problems of the Volterra integro-differential equation type. singular problems are considered in $[2,3,5,7]$.

In $\mathrm{Na}$ [8], ordinary differential equations with two point boundary conditions are solved by a method of parallel shooting. The general idea here was to divide the interval of integration into a number of subintervals and to find approximations to the solution of the differential equation on each subinterval. Continuity of the solution from subinterval to subinterval was imposed. In [11], this method was extended for application to second order two point boundary value problems of the Volterra type. In contrast to this approach, which involves expressing the problem as an initial value problem, other approaches such as that in [4] for linear problems and in [9] for nonlinear problems exist. In this article, the parallel shooting method in [11] is reworked for application to problems which have at most a finite number of singularities in the kernel. A numerical example illustrates the procedure and the reader is able to compare the results of this indirect method of solution with the work in [5].

\section{NUMERICAL METHODS}

Let $I_{N}=\left\{x_{k}=k h, k=0,1, \ldots, n, n=2^{N}, h>0, n h=a\right\}$ and $J_{M}=\left\{c_{k}=k a / m, k=0,1, \ldots, m, m=2^{M}, M \leq N\right\}$. The methods to be considered are comprised of three distinct parts: $(\rho, \sigma)$ denotes a pair of polynomials which characterize a linear multistep method for numerically solving a second order ordinary differential equation; $\mathbf{Q}$ will denote a set of quadrature weights associated with the numerical integration of the singular integrand; and PS will denote a parallel shooting method associated with solving a Volterra integro-differential problem with its boundary conditions.

In particular, to establish the method $(\rho, \sigma)$ let $\rho(z)=\sum_{i=0}^{k} \alpha_{\imath} z^{2}, \sigma(z)=\sum_{i=0}^{k} \beta_{\imath} z^{2}$ and for a problem of the form $y^{\prime \prime}=\varphi(x, y)$, we write

$$
\sum_{\imath=0}^{k} \alpha_{\imath} y_{\ell+\imath}=h^{2} \sum_{\imath=0}^{k} \beta_{\imath} \varphi_{\ell+\imath}, \quad \ell=0,1, \ldots, n-k .
$$

Here $y_{\imath}$ denotes an approximation to $y\left(x_{\jmath}\right)$ and $\varphi_{\jmath}=\varphi\left(x_{\jmath}, y_{\jmath}\right)$.

To develop quadrature rules for the approximation of an integral with a singular integrand, consider the integral

$$
\int_{0}^{x} \Psi(t) g(t) d t
$$

where $g$ is assumed to be continuous on $[0, a]$ and $\psi$ is assumed to have at most a finite number of singularities on $[0, a]$ but can be simply integrated with respect to all polynomials of all degrees. Thus

$$
\int_{0}^{x_{\jmath}} \Psi(t) g(t) d t \doteq \sum_{\imath=0}^{j} w_{\imath} g\left(x_{\imath}\right)
$$

where 


$$
w_{\mathfrak{n}}=\int_{0}^{x_{\jmath}} \widehat{g}_{\imath}(t) \Psi(t) d t
$$

with

$$
\widehat{g}_{\imath}(t)=\frac{R_{j}(t) g\left(x_{\imath}\right)}{\left(t-x_{\imath}\right) R_{\jmath}\left(x_{\imath}\right)}, R_{\jmath}(t)=\prod_{\imath=0}^{J}\left(t-x_{\imath}\right) .
$$

For each $j, j=k, k+1, \ldots, n$, a set of quadrature weights is determined. For the integrand (2), these are collectively denoted by $Q$.

The third part of the method is a common method of solution for nonlinear ODEs with two-point boundary conditions known as the shooting method. In particular, a parallel shooting method is implemented. To describe a parallel shooting method (PS), the equation (1) is rewritten as a system of first order equations

$$
\begin{aligned}
& d y / d x=u \\
& d u / d x=f(x, y, z) .
\end{aligned}
$$

To express (5) as an initial value problem, we have $y(0)=A$ and we set $u(0)=s$, where $s$ represents the initial slope and is unknown. On each subinterval $\left[c_{2}, c_{2+1}\right], c_{2} \in \mathbf{J}_{m}$, an initial value problem is defined by

$$
\begin{aligned}
d y^{(2+1)} / d x & =u^{(2+1)} \\
d u^{(2+1)} / d x & =f\left(x, y^{(2+1)}, z^{(2+1)}\right), i=0, \ldots, m-1
\end{aligned}
$$

with initial conditions on each subinterval given by $y^{(2+1)}\left(c_{2}\right)=C_{2}$ and $u^{(2+1)}\left(c_{2}\right)=D_{2}$. In particular, $C_{o}=A, D_{o}=s$ and $C_{m}=B$. At each point $c_{1}, i=1,2, \ldots, m-1$, the following continuity conditions are assumed:

$$
\begin{gathered}
y^{(\imath)}\left(c_{2}\right)=y^{(2+1)}\left(c_{2}\right)=C_{2} \\
u^{(i)}\left(c_{2}\right)=u^{(\imath+1)}\left(c_{2}\right)=D_{2}, i=1,2, \ldots, m-1 .
\end{gathered}
$$

In general, the problem is to determine $s, C_{\imath}, D_{\imath}, i=1,2, \ldots, m-1$ These conditions are expressed as:

$$
\begin{aligned}
\phi_{1}\left(D_{0}, C_{1}\right) & =y^{(1)}\left(c_{1}, D_{0}\right)-C_{1}=0 \\
\phi_{2}\left(D_{0}, D_{1}\right) & =u^{(1)}\left(c_{1}, D_{0}\right)-D_{1}=0 \\
\phi_{3}\left(D_{1}, C_{1}, C_{2}\right) & =y^{(2)}\left(c_{2}, D_{1}, C_{1}\right)-C_{2}=0 \\
& \vdots \\
\phi_{2 m-3}\left(D_{m-2}, C_{m-2}, C_{m-1}\right) & =y^{(m-1)}\left(c_{m-1}, D_{m-2}, C_{m-2}\right)-C_{m-1}=0 \\
\phi_{2 m-2}\left(D_{m-2}, C_{m-2}, D_{m-1}\right) & =u^{(m-1)}\left(c_{m-1}, D_{m-2}, C_{m-2}\right)-D_{m-1}=0 \\
\phi_{2 m-1}\left(D_{m-1}, C_{m-1}\right) & =y^{(m)}\left(c_{m}, D_{m-1}, C_{m-1}\right)-C_{m}=0 .
\end{aligned}
$$

Each unknown is determined iteratively by first expanding $\phi_{i}$ in a Taylor expansion through linear terms with respect to each argument. The initial approximations are denoted by $D_{o}^{1}, C_{\imath}^{1}, D_{\imath}^{1}, i=1,2, \ldots, m-1$ The system is given by

$$
A^{2} \Delta Z^{2}=-\Psi^{2}
$$

where $\Delta Z^{2}=Z^{2+1}-Z^{2}, Z^{2}=\left(D_{0}^{2}, C_{1}^{2}, D_{1}^{2}, \ldots, D_{m-1}^{2}\right)^{T}, \Psi^{2}=\left(\Psi_{1}^{2}, \ldots, \Psi_{2 m-1}^{2}\right)^{T}$ and $A^{2}$ is a four band matrix with elements given by

$$
\begin{aligned}
a_{k \cdot k-1}^{2} & =\partial y^{[(k-1) / 2]+1} / \partial C_{(k-1) / 2} \\
a_{k \cdot k}^{2} & =\partial y^{[(k-1) / 2]+1} / \partial D_{(k-1) / 2} \\
a_{k k+1}^{2} & =-1 \\
a_{k \cdot k+2}^{2} & =0
\end{aligned}
$$

for $k$ odd, $k=3(2) 2 m-3$ and 


$$
\begin{aligned}
a_{k \cdot k-2}^{2} & =\partial u^{(k / 2)} / \partial C_{(k / 2)-1} \\
a_{k \cdot k-1}^{2} & =\partial u^{(k / 2)} / \partial D_{(k / 2)-1} \\
a_{k \cdot k}^{2} & =0 \\
a_{k \cdot k+1}^{2} & =-1
\end{aligned}
$$

for $k$ even, $k=4(2) 2 m-2$. For the remaining values, we have

$$
\begin{aligned}
a_{1 \cdot 1}^{2} & =\partial y^{(1)} / \partial D_{0} \\
a_{1 \cdot 2}^{2} & =-1 \\
a_{1 \cdot k}^{2} & =0, k=3(1) 2 m-1 \\
a_{2 \cdot 1}^{2} & =\partial u^{(1)} / \partial D_{0} \\
a_{2 \cdot 2}^{2} & =0, a_{2 \cdot 3}^{2}=-1, a_{2 \cdot k}^{2}=0, k=4(1) 2 m-1 \\
a_{2 m-1 \cdot 2 m-2}^{2} & =\partial y^{(m)} / \partial C_{m-1} \\
a_{2 m-1 \cdot 2 m-1}^{2} & =\partial y^{(m)} / \partial D_{m-1} \\
a_{2 m-1 \cdot k}^{2} & =0, k=1(1) 2 m-3 .
\end{aligned}
$$

To obtain values for the partial derivatives, the following notations are introduced

$$
\begin{aligned}
Y_{D_{i}} & =\partial y^{(i+1)} / \partial D_{i}, i=0,1, \ldots, m-1 \\
U_{D_{1}} & =\partial u^{(i+1)} / \partial D_{i} \\
Y_{C_{i}} & =\partial y^{(i+1)} / \partial C_{i}, i=1,2, \ldots, m-1 \\
U_{C_{i}} & =\partial u^{(i+1)} / \partial C_{i} .
\end{aligned}
$$

Differentiating the systems (8) with respect to each unknown gives rise to a system of integrodifferential equations:

$$
\begin{aligned}
& \partial Y_{D,} / \partial x=U_{D_{1}} \\
& \partial U_{D_{1}} / \partial x=\partial f(x, y(x), z(x)) / \partial D_{\imath} \\
& \partial Y_{C_{1}} / \partial x=U_{C_{1}} \\
& \partial U_{C_{\imath}} / \partial x=\partial f(x, y(x), z(x)) / \partial C_{\imath} \\
& Y_{D_{1}}=0, U_{D_{1}}=1 \\
& i=0,1, \ldots, m-1 \\
& Y_{C_{1}}=1, U_{C_{1}}=0 \\
& i=1,2, \ldots, m-1
\end{aligned}
$$

These $2 m-1$ systems of first order integro-differential equations can be solved on their respective subintervals.

1 Let $\ell=0$ and assume a first approximation to the unknown values as follows:

$$
\begin{aligned}
& D_{i}^{\ell}=(y(a)-y(0)) / m N, i=0,1, \ldots, m-1 \\
& C_{\imath}^{\ell}=y(0)+i(y(a)-y(0)) / m, i=0,1, \ldots, m
\end{aligned}
$$

where $N$ is the number of partition points between $c_{i}$ and $c_{2+1}$ for each $i=0,1, \ldots, m-1$.

2. Set $\ell=\ell+1$ and integrate the systems of the first order integro-differential equation (6), taking $D_{\imath}=D_{\imath}^{\ell}$ and $C_{\imath}=C_{\imath}^{\ell}$.

3. Integrate the systems of integro-differential problems given by the system (9)

4. Using the values obtained in steps 2 and 3 , solve the system (7) to obtain $C_{2}^{\ell+1}, i=0,1, \ldots, m$ and $D_{\imath}^{\ell+1}, i=0,1, \ldots, m-1$. If the desired accuracy between successive iterations is reached, then stop. Otherwise, repeat starting with step 2 .

\section{THEORETICAL RESULTS}

In this section we consider the convergence of the method. Let $(\rho, \sigma)$ denote the characteristic polynomials associated with the method for solving a first order differential equation and let $\mathbf{Q}$ denote the quadrature rule

The polynomial $\rho(z)$ is given by $\rho(z)=\alpha_{k} z^{k}+\ldots+\alpha_{0} \quad$ Let $\gamma_{2}, i=0,1,2, \ldots$ be a set of coefficients defined by 


$$
1 /\left(\alpha_{k}+\alpha_{k-1} z+\ldots+\alpha_{0} z^{k}\right)=\gamma_{0}+\gamma_{1} z+\gamma_{2} z^{2}+\ldots .
$$

DEFINITION 1. The linear $k$-step methods for first order ODEs and defined by the polynomials $(\rho, \sigma)$ is said to be zero-stable if no root of $\rho$ has modulus greater than one and that roots of modulus one be simple

Let $Y\left(x_{n}\right)$ denote the exact solution of system (5) and $F\left(x_{n}, Y\left(x_{n}\right), z\left(x_{n}\right)\right)=\left(u\left(x_{n}\right), f\left(x_{n}, y\left(x_{n}\right), z\left(x_{n}\right)\right)\right)^{T}$ at $x n \in I_{N}$.

DEFINITION 2. Denote the two part method of solution by $((\rho, \sigma), \mathbf{Q})$. The difference operators $L$ and $M$ (notation taken from [1]) associated with the method are defined by

$$
L\left[Y\left(x_{n}\right) ; h\right]=\sum_{\imath=0}^{k}\left(\alpha_{i} Y\left(x_{n+1}\right)=h \beta_{\imath} Y^{\prime}\left(x_{n+\imath}\right)\right), \quad x_{n} \in I_{N}
$$

and

$$
M\left[Y\left(x_{n}\right) ; h\right]=\sum_{i=0}^{k}\left(\alpha_{2} Y\left(x_{n+2}\right)-h \beta_{2} F\left(x_{n+2}, Y\left(x_{n+\imath}\right), \widehat{z}\left(x_{n+\imath}\right)\right)\right)
$$

where

$$
\widehat{z}\left(x_{n+\imath}\right)=\sum_{\jmath=0}^{n+2} \omega_{n_{1+1}, j} G\left(t_{\jmath}, y\left(t_{\jmath}\right)\right)
$$

Assuming $Y(x)$ is at least $p+1$ times differentiable, then $L\left[Y\left(x_{n}\right) ; h\right]$ can be expanded about the point $x_{n}$ to give

$$
L\left[Y\left(x_{n}\right) ; h\right]=C_{0} Y\left(x_{n}\right)+C_{1} Y^{(1)}\left(x_{n}\right)+\ldots
$$

where $\left\{C_{\imath}\right\}$ are coefficients independent of the function. We define the order of $L$ to be the unique integer $p$ such that $C_{\imath}=0, i=0(1) p$ but $C_{p+1} \neq 0$.

For sufficiently smooth functions $F$, the two operators $L$ and $M$ are related by

$$
M\left[Y\left(x_{n}\right) ; h\right]=L\left[Y\left(x_{n}\right) ; h\right]-h \sum_{\imath=0}^{k} \beta_{\imath} \frac{\partial F\left(x_{n+\imath}, Y\left(x_{n+\imath}\right), z_{n+2}^{*}\right)}{\partial z} E_{n+\imath}^{*}
$$

where

$$
z_{n+2}^{*} \in \operatorname{INT}\left(\widehat{z}\left(x_{n+2}\right), z\left(x_{n+2}\right)\right) \text { and } E_{n+2}^{*}=\left(0, \widehat{z}\left(x_{n+2}\right)-z_{n+2}\right)^{T} .
$$

For the quadrature $Q$, we assume that weights $\omega_{n z}$ exist which for, all $f \in C[0, a]$ and for all $0 \leq x \leq a$, satisfy the conditions:

i) $\left|\int_{0}^{x} S(x, t) f(t) d t-\sum_{i=0}^{n} \omega_{n \imath} f\left(x_{\imath}\right)\right| \rightarrow 0$

as $h \rightarrow 0, n \rightarrow \infty$ such that $n h=x$. In addition, we assume that $\max \left|\omega_{k_{2}}\right| \rightarrow 0$, as $h \rightarrow 0$, $k \rightarrow \infty, k h=x_{k}$ where the maximum is over $0 \leq i \leq k$ and $0 \leq k \leq n$; and

ii) $[0, a]$ can be partitioned into a finite number of subintervals with $0=z_{0}<z_{1}<\ldots<z_{n}=a$ such that for any $k \leq n$, the sum of the absolute values of the weights $\omega_{k z}$ associated with points in each subinterval $\left[z_{2}, z_{\imath+1}\right]$ can be made as small as possible. Thus for a given $h$ sufficiently small and any $\epsilon>0$ there exists a partition of $[0, a]$ such that for $\left[z_{2}, z_{\imath+1}\right]$ partitioned by $x_{n_{1}}, \ldots, x_{n_{1+1}}-1$

$$
\sum_{\jmath=n_{1}}^{n_{3+1}-1}\left|\omega_{h_{n+1}-1}\right|<\epsilon
$$

With the above assumptions, we now define the order of the quadrature. 
DEFINITION 3. Let $f(t) \in C^{q}[0, a], q>0$. The quadrature rule $Q$ will be said to have order $q$ if $q$ is the largest positive real number such that

$$
\left|\int_{0}^{x_{k}} S\left(x_{k}, t\right) f(t) d t-\sum_{i=0}^{k} \omega_{k \imath} f\left(x_{\imath}\right)\right| \leq C h^{q}, C<\infty, \forall x_{k} \in I_{N} .
$$

For special forms of $S(x, t)$, the order may be non-integer (see, [2])

For the parallel shooting method, starting values are required on each subinterval of the partition $\mathbf{J}_{\mathbf{M}}$ The two part method is likewise employed on each subinterval.

DEFINITION 4. The linear two part method $((\rho, \sigma), \mathbf{Q})$ is said to be convergent if for all equations of the form (5) subject to the conditions stated on $f$ and $K$, we have that

i) $\lim _{\substack{h \rightarrow 0 \\ n h=x}} Y_{n}=Y(x)$ and

ii) for all solutions $\left\{Y_{n}\right\}$ of (3) and (4) satisfying the starting conditions $Y_{J}=Y_{J}(h)$ for which $\lim _{h \rightarrow 0} Y_{\jmath}(h)=Y_{0}, j=0(1) k-1$ and $z_{j}$ for which $\lim _{h \rightarrow 0} z_{\jmath}=0, j=1(1) k-1$.

REMARK. It is well known [6, p. 218], that zero-stability of $(\rho, \sigma)$ is a necessary condition for convergence.

For the function, $F(x, Y, z)$ the following notation is introduced

i) $\quad F_{n}=F\left(x_{n}, Y_{n}, z_{n}\right)$

ii) $\quad \widehat{F}=F\left(x_{n}, Y\left(x_{n}\right), \sum_{i=0}^{n} \omega_{n i} K\left(x_{n}, x_{\imath}, y\left(x_{\imath}\right)\right)\right)$

and

iii) $\widetilde{F}=F\left(x_{n}, Y\left(x_{n}\right), \sum_{i=0}^{n} \omega_{n 2} K\left(x_{n}, x_{2}, y_{2}\right)\right)$.

Then from

$$
\sum_{\imath=0}^{k} \alpha_{\imath} Y_{n+\imath}-h \sum_{\imath=0}^{k} \beta_{\imath} F_{n+\imath}=0
$$

subtract the same equation with approximate values replaced by exact values to get

$$
M_{n h}=\sum_{\imath=0}^{k}\left(\alpha_{\imath} E_{n+\imath}-\beta_{\imath}\left(F_{n+\imath}-\widehat{F}\right)\right)
$$

where $E_{n}=Y_{n}-Y\left(x_{n}\right)$. Further, we write $F_{n}-\widehat{F}_{n}=D_{n}^{(1)}+D_{n}^{(2)}$ where $D_{n}^{(1)}=\left(d_{1 n}^{(1)}, d_{2 n}^{(1)}\right)^{T}=F_{n}-\widetilde{F}_{n}$ and $D_{n}^{(2)}=\left(0, d_{2 n}^{(2)}\right)^{T}=\widetilde{F}_{n}-\widehat{F}_{n}$. We define $G_{n}=\left(g_{1 n}, g_{2 n}\right)^{T}$ where

$$
g_{1 n}=\left(\begin{array}{ccc}
d_{1 n}^{(1)}\left(e_{n}^{\prime}\right)^{-1} & \text { if } & e_{n}^{\prime} \neq 0 \\
0 & \text { if } & e_{n}^{\prime}=0
\end{array}\right.
$$

and

$$
g_{2 n}=\left(\begin{array}{ccc}
d_{2 n}^{(2)} e_{n}^{-1} & \text { if } & e_{n} \neq 0 \\
0 & \text { if } & e_{n}=0
\end{array}\right.
$$

Then $\left\|G_{n}\right\| \infty<L_{F 1}=\max \left(L_{1}, 1\right)$. Similarly, define $R_{n}=\left(r_{1 n}, r_{2 n}\right)^{T}$ by

$$
\begin{aligned}
& r_{1 n}=0 \\
& r_{2 n}= \begin{cases}d_{2 n}^{(2)}\left(\sum_{\imath=0}^{n}\left|e_{\imath}\right|\right)^{-1} & \text { if } \sum_{i=0}^{n}\left|e_{\imath}\right| \neq 0 \\
0 & \text { if } \sum_{i=0}^{n}\left|e_{\imath}\right|=0\end{cases}
\end{aligned}
$$


Then $\left\|R_{n}\right\|_{\infty} \leq L_{F 2} L_{3} W$ where $L_{F 2}=\max \left(L_{2}, 1\right)$ and $\left|\omega_{n 2}\right| \leq W$ for all $0 \leq i \leq N$

With these notations, we can now write down a difference equation for the errors $\left\{E_{z}\right\}$

$$
\sum_{\imath=0}^{k} \alpha_{\imath} E_{m+\imath}=h \sum_{\imath=0}^{k} \beta_{i} g_{m+\imath} E_{m+\imath}+\sum_{\imath=0}^{k} \beta_{\imath} d_{m+\imath} \sum_{\jmath=0}^{m+\imath} \omega_{m+\imath \cdot j} E_{\jmath}+M_{m h} .
$$

To establish the convergence of this sequence (i.e. $E_{\imath} \rightarrow 0$ as $i \rightarrow \infty, h \rightarrow 0$ with $i h=x_{\imath}$ ), let us recall a lemma from Linz [7, p 368]:

LEMMA 1. If

$$
\begin{gathered}
\left|\epsilon_{k}\right| \leq \sum_{i=0}^{k-1}\left|\alpha_{k_{2}}\right|\left|\epsilon_{\imath}\right|+T \text { for } k=r, r+1, \ldots, T>0 \\
\quad\left|\epsilon_{k}\right| \leq \eta, k=0(1) r-1 \\
\sum_{i=0}^{k-1}\left|\alpha_{k_{2}}\right| \leq \alpha<1 \text { for } k=r, r+1, \ldots
\end{gathered}
$$

then

$$
|\epsilon| \leq \frac{T+\alpha \eta}{1-\alpha}, k=r, r+1, \ldots
$$

THEOREM 1. Let us assume that the kernel $S(x, t) G(t, y(t))$ satisfies the conditions stated in section 1 and that the weights $\left\{\omega_{n \imath}\right\}$ exist which for all $G(t, y(t)) \in C[0, a]$ and for all $0 \leq x \leq a$, satisfy conditions i) and ii) of this section. Let $\rho(z)=\alpha_{k} z^{k}+\ldots+\alpha_{0}$ satisfy the condition of zero-stability and assume the sequence $\left\{\gamma_{2}\right\}$ is such that $\sup \left|\gamma_{2}\right|=\Gamma<\infty$. Let $\tau, T^{*}, \beta, B^{*}$ and $\Lambda$ be constants such that $\tau_{m \imath}=\beta_{\imath} d_{m+1}$ and $\left|\tau_{m \imath}\right|<\tau, T^{*}=k \tau, \beta_{\imath, m}=\beta_{\imath} g_{m+\imath}$ and $\sum_{\imath=0}^{k}\left|\beta_{\imath, m}\right| \leq B^{*}$, and $\left|M_{n h}\right|<\Lambda$ for all $0 \leq n \leq N$ Then every solution of

$$
\sum_{\imath=0}^{k} \alpha_{\imath} E_{m+\imath}=h \sum_{j=0}^{k} \beta_{\imath, m+\imath} E_{m+\imath}+h \sum_{\imath=0}^{k} \tau_{m \imath} \sum_{j=0}^{n+2} \omega_{m+\imath \cdot j} E_{\jmath}+M_{m h}
$$

for which $\left\|E_{\imath}\right\|<E, i=0(1) k-1$ satisfies $\left\|E_{n}\right\|=O\left(E^{*}\right) \forall x_{n} \in I_{N}$ where

$$
E^{*}=\frac{(N \Gamma \Lambda+A \Gamma E k)}{1-h \Gamma B^{*}-h \Gamma T^{*}\left|\omega_{n n}\right|}
$$

and $A=\sum_{i=0}^{k}\left|\alpha_{i}\right|$.

PROOF. For $j=0(1) n-k$, multiply equation (11) corresponding to $m=n-k-j$ by $\gamma$, and add the resulting equations. On the left hand side, we get $E_{n}$ plus terms, where we have used the fact that $\alpha_{k} \gamma_{0}=1$ and the terms are all zero except for those involving $E_{m}, m=0(1) k-1$. Each of these non zero terms is bounded by $k A E \Gamma$.

On the right hand side, we get

$$
\begin{aligned}
h\left(\beta_{k, n-k} \gamma_{0} E_{n}+\left(\beta_{k, n-k-1} \gamma_{1}+\beta_{k-1, n-k} \gamma_{0}\right) E_{n-1}+\ldots+\left(B_{1,0} \gamma_{n-k}+B_{0,1} \gamma_{n-k-1}\right) E_{1}+\beta_{0,0} \gamma_{n-k} E_{0}\right) \\
+h\left(\gamma_{0} \tau_{k, n-1} \sum_{\imath=0}^{n} \omega_{n \imath} E_{\imath}+\left(\gamma_{0} \tau_{k-1, n-k-1}+\gamma_{1} \tau_{k, n-k-1}\right) \sum_{\imath=0}^{n-1} \omega_{n-1, \imath} E_{\imath}\right. \\
\left.\quad+\ldots+\left(\gamma_{n-k} \tau_{1,1}+\gamma_{n-k-1} \tau_{0,1}\right) \sum_{\imath=0}^{1} \omega_{1 \imath}, E_{\imath}+\gamma_{n-k} \tau_{0,0} \omega_{00} E_{0}\right)+\gamma_{0} \lambda_{n-k}+\ldots+\gamma_{n-k} \lambda_{0} .
\end{aligned}
$$

Taking norms and applying the bounds in the hypothesis leads to

$$
\left\|E_{n}\right\| \leq h \Gamma B^{*} \sum_{i=0}^{n}\left\|E_{\imath}\right\|+h T^{*} \Gamma \sum_{i=0}^{n} \sum_{\jmath=0}^{\imath}\left|\omega_{\imath \jmath}\right|\left\|E_{\jmath}\right\|+N \Gamma \Lambda+A \Gamma E k .
$$


Reorganizing the terms in the double sum leads to

$$
\left\|E_{n}\right\| \leq h \Gamma B^{*} \sum_{i=0}^{n}\left\|E_{\imath}\right\|+h T^{*} \Gamma \sum_{i=0}^{n}\left(\left|\omega_{n \imath}^{\prime}\right|\left\|E_{\imath}\right\|\right)+N \Gamma \Lambda+A \Gamma E k
$$

where

$$
\left|\omega_{n j}^{\prime}\right|=\sum_{i=j}^{n}\left|\omega_{\imath \jmath}\right|, \quad j=0(1) n
$$

As a final step in organizing this equation, the terms involving $\left\|E_{n}\right\|$ are collected and we can write

$$
\left\|E_{n}\right\| \leq h M^{*} \sum_{i=0}^{n-1}\left\|E_{\imath}\right\|+h N^{*} \sum_{i=0}^{N-1}\left|\omega_{n 2}^{\prime}\right|\left\|E_{\imath}\right\|+E^{*}
$$

where

$$
X=1-h \Gamma B^{*}-h \Gamma T^{*}\left|\omega_{n n}^{\prime}\right|, \quad M^{*}=\Gamma B^{*} / X, N^{*}=\Gamma T^{*} / X \text { and } E^{*}=(N \Gamma \Lambda+A \Gamma E k) / X .
$$

Note that $\left|\omega_{n n}\right|=\left|\omega_{n n}^{\prime}\right|$.

By assumption and for $h$ sufficiently small, the interval $[0, a]$ can be divided into a finite number of subintervals such that $h M^{*}+h N^{*}<1$ for all $n \leq N$ and $n_{k-1}<n$ Thus for all points $x_{n}$ in the first subinterval

$$
\left\|E_{n}\right\| \leq \frac{E^{*}}{\left(1-h\left(M^{*}+N^{*}\right)\right)}
$$

which follows from Lemma 1 . For points in the second subinterval

$$
\left\|E_{n}\right\| \leq h \sum_{\imath=0}^{n_{1}-1}\left(M^{*}+N^{*}\left|\omega_{n_{1}}\right|\right)\left\|E_{\imath}\right\|+h \sum_{\imath=n_{1}}^{n-1}\left(M^{*}+N^{*}\left|\omega_{n 2}\right|\right)\left\|E_{\imath}\right\|+E^{*}
$$

Substituting into the first sum the bound for points in the first subinterval and again applying Lemma 1 gives

$$
\left\|E_{n}\right\|=O\left(E^{*}\right) .
$$

Since there are only a finite number of subintervals, by induction we conclude that

$$
\left\|E_{n}\right\|=O\left(E^{*}\right) \forall x_{n} \epsilon I_{N} .
$$

REMARK. For special forms of the function $S(x, t)$, sharper expreśsions for the error bound are possible (see, [2])

\section{NUMERICAL EXAMPLE}

This section contains a numerical example taken from [5]. The particular method which was employed to solve the problem is described below. Table 2 contains some numerical results

EXAMPLE:

$$
\begin{aligned}
y^{\prime \prime}=\frac{-y^{2}}{2 \sqrt{x+1}} & +\frac{5+2 x}{4(1+x)^{\frac{5}{2}}}-\left\{\frac{2(1+x)^{\frac{3}{2}}-1}{3}+(1+x) \times\right. \\
& \ln (2 x+1+2 \sqrt{x(x+1)})\}+\int_{0}^{x}\left(\frac{x+1}{\sqrt{t}}+1+t\right) y(t) d t
\end{aligned}
$$

with the boundary conditions $y(0)=1$ and $y(1)=\frac{1}{\sqrt{2}} \quad$ The exact solution for this example is $y(x)=\frac{1}{\sqrt{1+x}}$

METHOD: For the system (6), the Milne-Simpson method defined by $(\rho, \sigma)$ where $\rho(z)=z^{2}-1$ and $\sigma(z)=\left(z^{2}+4 z+1\right) / 3$ is used to discretize each equation. Since the integrand in the example is such that part can be solved by usual quadrature rules and the other by product integration rules, then the 
weights of the fourth order Newton-Gregory rules (see Table 1) are used for the former and a set of product integration rules for the latter based on Simpson's product integration with weights $\left\{\omega_{k j}\right\}$ The weights $\left\{\omega_{k j}\right\}$, for $k$ even, consist of a composite Simpson product integration rule with weights given by

$$
\begin{aligned}
\omega_{k \jmath} & =.5 S_{0}(j, k)-1.5 S_{1}(j, k)+S_{2}(j, k) \\
\omega_{k \jmath+1} & =S_{0}(j, k)+2.0 S_{1}(j, k) \\
\omega_{k \jmath+2} & =.5 S_{0}(j, k)-0.5 S_{1}(j, k)
\end{aligned}
$$

for $j=0(2) k$ where

$$
\begin{aligned}
& S_{0}(\jmath, k)=\left(1 / h^{2}\right) \int_{x,}^{x_{j+2}}\left(t-t_{\jmath}\right)^{2} S\left(x_{k}, t\right) d t \\
& S_{1}(j, k)=(1 / h) \int_{x,}^{x_{j+2}}\left(t-t_{\jmath}\right) S\left(x_{k}, t\right) d t
\end{aligned}
$$

and

$$
S_{2}(j, k)=\int_{x_{\jmath}}^{x_{\jmath+2}} S\left(x_{k}, t\right) d t
$$

For $k$ odd, the above rule is used on each subinterval $\left[x_{\jmath}, x_{\jmath+2}\right]$ for $j=0(2) k-3$ and the four weights obtained by using the Lagrange three step rule on $\left[x_{k-3}, x_{k}\right]$ to integrate $S\left(x_{k}, t\right)$ are given by

$$
\begin{aligned}
& \omega_{k-3 k}^{\prime}=(-1 / 6) S_{0}^{\prime}(k-3, k)+S_{1}^{\prime}(k-3, k)-(11 / 6) S_{2}^{\prime}(k-3, k)+S_{3}^{\prime}(k-3, k) \\
& \omega_{k-2 k}^{\prime}=(1 / 2) S_{0}^{\prime}(k-3, k)-(5 / 2) S_{1}^{\prime}(k-3, k)+3 S_{2}^{\prime}(k-3, k) \\
& \omega_{k-1 k}^{\prime}=(-1 / 2) S_{0}^{\prime}(k-3, k)+(3 / 2) S_{1}^{\prime}(k-3, k)-S_{2}^{\prime}(k-3, k)
\end{aligned}
$$

and

$$
w_{k k}^{\prime}=(1 / 6) S_{0}^{\prime}(k-3, k)-(1 / 2) S_{1}^{\prime}(k-3, k)+(1 / 3) S_{2}^{\prime}(k-3, k)
$$

where

$$
\begin{aligned}
& S_{0}^{\prime}(k-3, k)=\left(1 / h^{3}\right) \int_{x_{k-3}}^{x_{k}}\left(t-t_{k-3}\right)^{3} S\left(x_{k}, t\right) d t \\
& S_{1}^{\prime}(k-3, k)=\left(1 / h^{2}\right) \int_{x_{k-3}}^{x_{k}}\left(t-t_{k-3}\right)^{2} S\left(x_{k}, t\right) d t \\
& S_{2}^{\prime}(k-3, k)=(1 / h) \int_{x_{k-3}}^{x_{k}}\left(t-t_{k-3}\right) S\left(x_{k}, t\right) d t
\end{aligned}
$$

and

$$
S_{3}^{\prime}(k-3, k)=\int_{x_{k-3}}^{x_{k}} S\left(x_{k}, t\right) d t
$$

Note that for the system (2) there are special weights required for $\left\{v_{\imath \jmath}\right\}$ and $\left\{\omega_{\imath \jmath}\right\}$ to approximate the system over $\left[0, x_{1}\right]$. In particular a system of four equations is solved to find approximations for $y_{1}, y_{2}, u_{1}$, and $u_{2}$ as follows

$$
\begin{aligned}
& y_{1}-y_{0}=\frac{h}{12}\left(5 u_{0}+8 u_{1}-u_{2}\right) \\
& u_{1}-u_{0}=\frac{h}{12}\left(5 f_{0}+8 f_{1}-f_{2}\right) \\
& y_{2}-y_{0}=\frac{h}{3}\left(u_{0}+4 u_{1}+u_{2}\right) \\
& u_{2}-u_{0}=\frac{h}{3}\left(f_{0}+4 f_{1}+f_{2}\right) .
\end{aligned}
$$

The weights $\left\{v_{\imath j}\right\}$ are given by $\left\{\frac{5}{12}, \frac{8}{12}, \frac{-1}{12}\right\}$ while for the weights $\left\{\omega_{\imath \jmath}\right\}$, suffice it to say that the second degree Lagrange polynomial interpolating at $x=x_{0}, x_{1}, x_{2}$ is analytically integrated as a product with 
$\frac{x+1}{\sqrt{t}}$ over $\left[0, x_{1}\right]$ The same method was used to solve each system of partial derivatives

Table 1: Newton-Gregory Quadrature Weights

\begin{tabular}{|c|c|c|c|c|c|c|c|}
\hline$n$ & $24 v_{n j}, \jmath=0(1) n$ \\
\hline 2 & 8 & 32 & 8 & & & & \\
\hline 3 & 9 & 27 & 27 & 9 & & & \\
\hline 4 & 9 & 28 & 22 & 28 & 9 & & \\
\hline 5 & 9 & 28 & 23 & 23 & 28 & 9 & \\
\hline 6 & 9 & 28 & 23 & 24 & 23 & 28 & 9 \\
\hline
\end{tabular}

Table 2. Table of Errors

\begin{tabular}{|l|l|l|}
\hline$x_{n}$ & $\left|e_{n}\right|(h=0.05)$ & $\left|e_{n}\right|(h=0.01)$ \\
\hline 0.2 & $4.67(-8)$ & $6.28(-9)$ \\
\hline 0.4 & $3.06(-7)$ & $4.07(-9)$ \\
\hline 0.6 & $3.32(-7)$ & $2.36(-9)$ \\
\hline 0.8 & $6.78(-8)$ & $6.89(-10)$ \\
\hline 1.0 & $1.55(-7)$ & $1.18(-9)$ \\
\hline
\end{tabular}

The method was applied using a tolerance of $h^{4}$ For $h=0.05$, four iterations were required to satisfy the tolerance and for $h=0.01$, the number of iterations was five. In [5], this problem was solved iteratively using a system approach to approximate the boundary value problem For a step $h=.05$, it took five iterations to satisfy the tolerance and there was a maximum error of $10^{-6}$

\section{REFERENCES}

[1] BRUNNER, $\mathrm{H}$. and LAMBERT, JD, Stability of numerical methods for Volterra integrodifferential equations, Computing 12 (1974), 75-89.

[2] deHOOG, F. and WEISS, R., Asymptotic expansions for product integration, Math. Comp. V.27 (1973), 295-306

[3] GAREY, L.E., Numerical methods for singular Volterra type equations with two-point boundary conditions, Intern. J. Computer Math. 32 (1990), 193-203.

[4] GAREY, L.E. and GLADWIN, C.J., Numerical methods for seccond order Volterra integrodifferential equations with two-point boundary conditions, Utilitas Máth. 35 (1989), 103-110

[5] GAREY, L.E. and SHAW, R.E., Numerical methods for singular non-linear Volterra type equations with two-point boundary conditions, Comp. \& Math. Appl. 26 (1993), 53-61

[6] HENRICI, P., Discrete Methods in Ordinary Differential Equations, John Wiley \& Sons, New York (1962).

[7] LINZ, P , Numerical methods for Volterra integral equations with singular kernels, SIAM J. Numer. Anal. 6 (1969), 365-374

[8] NA, T.Y., Computational Methods in Engineering Boundary Value Problems, Academic Press, New York (1979)

[9] SHAW, R.E and GAREY, L.E., Monotonic approximations for nonlinear second order Volterra integro-differential equations with two point boundary conditions, Utilitas Math. 38 (1992), 129136.

[10] SHAW, R.E. and GAREY, L.E., Vector processing an iterative method for nonlinear second order Volterra integro-differential equations with two-point boundary conditions, Congressus Numerantium (1992), 129-136.

[11] SHAW, R.E. and GAREY, L.E., A parallel shooting method for second order Volterra integrodifferential equations with two point boundary conditions, Intern. J. Comp. Math. 49 (1993), 61-66

[12] VOLK, W., The numerical solution of linear integro-differential equations by projection methods, J. Integral Equations 9 (suppl), (1985), 171-190. 


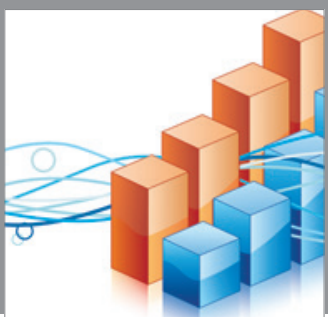

Advances in

Operations Research

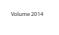

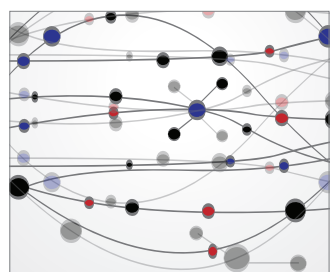

\section{The Scientific} World Journal
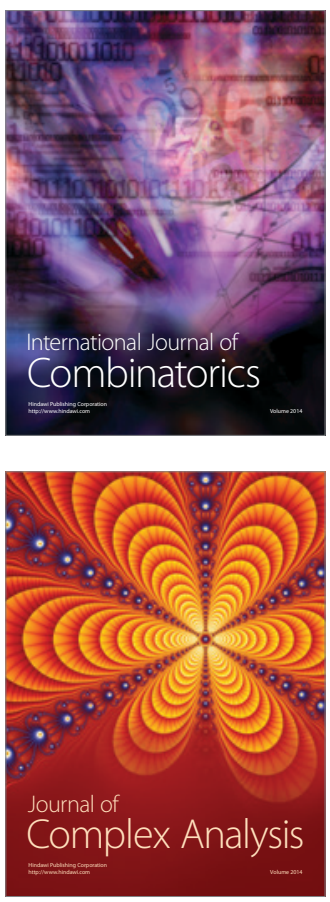

International Journal of

Mathematics and

Mathematical

Sciences
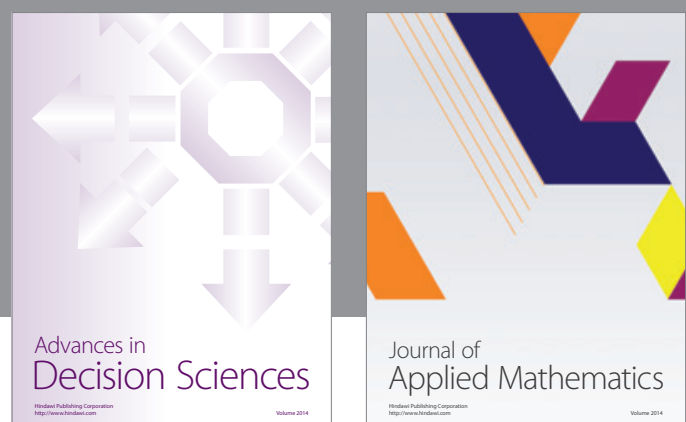

Journal of

Applied Mathematics
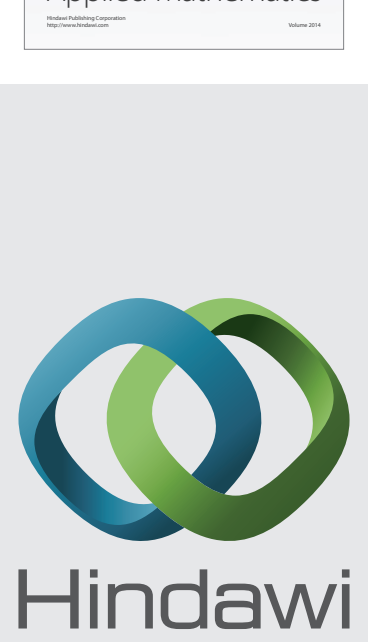

Submit your manuscripts at http://www.hindawi.com
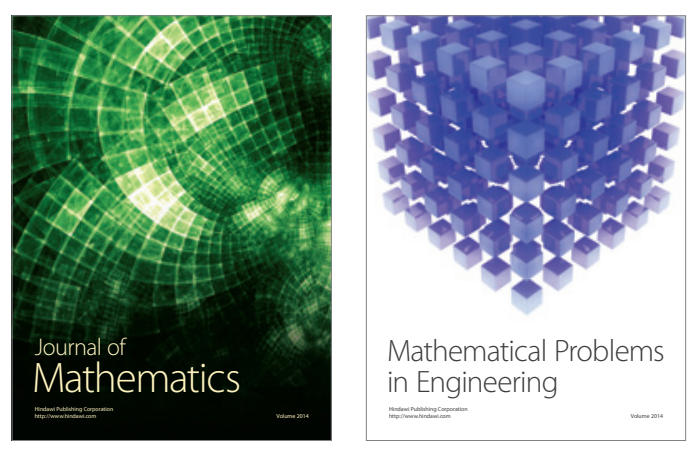

Mathematical Problems in Engineering
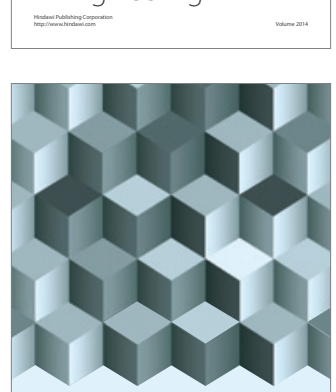

Journal of

Function Spaces
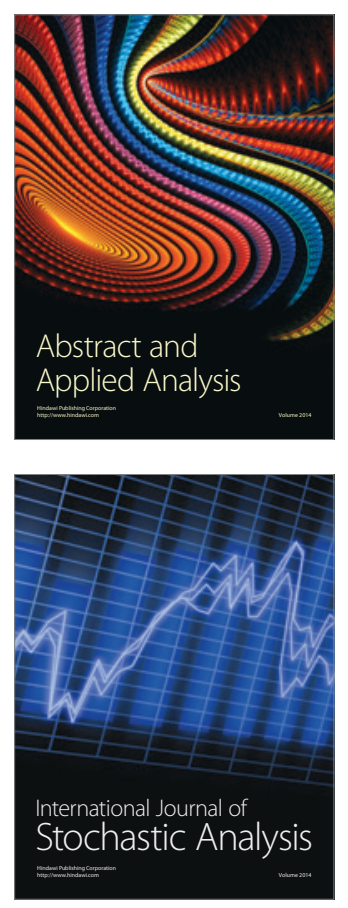

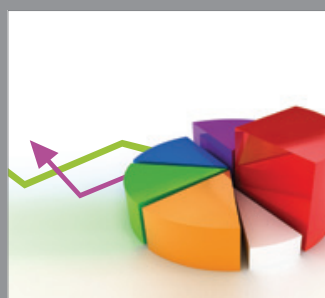

ournal of

Probability and Statistics

Promensencen
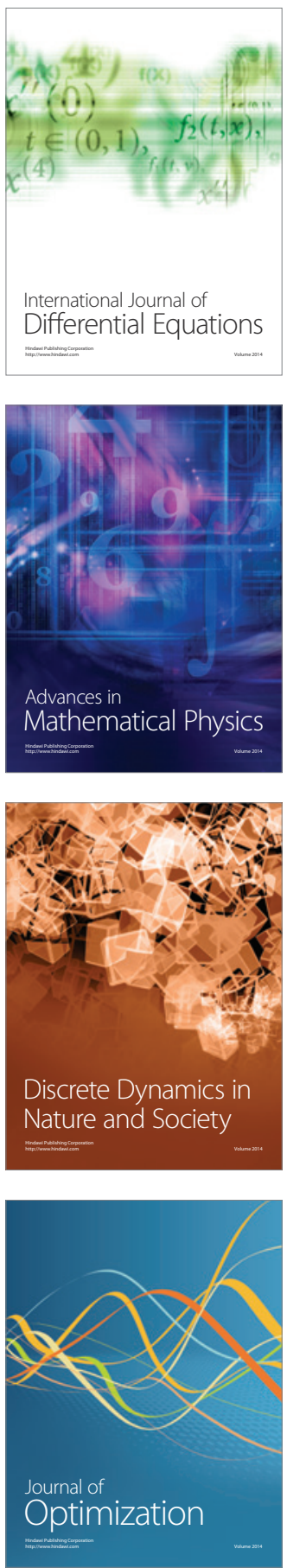